JoURnAL OF UNIVERSAL MATHEMATICS

Vol.4 No.1 PP.42-50 (2021)

ISSN-2618-5660

DOI: $10.33773 /$ jum.862564

\title{
THE NEW SET DIFFERENCE OPERATION BETWEEN INTERVAL VALUED INTUITIONISTIC FUZZY SETS
}

\author{
ARİF BAL AND GÖKHAN ÇUVALCIOĞLU
}

\begin{abstract}
In this paper, the new set difference is defined between intervalued intuitionistic fuzzy sets (IVIFSs).A different perspective is reduced to the formal set difference between IVIFSs. This new set difference $(-$.) between IVIFSs is introduced by using . operation. Instead of comparing this operator with all other set difference operations, the set difference operation $(-\cap)$ which is defined by using $\cap$ is used.

Some fundamental properties which are provided and not provided on $-\cap$ are examined whether satisfy or not satisfy on - operation. By the help of these examinations, it is seen that $-\cap$ and - . operations have different properties. The new properties about - . are studied.
\end{abstract}

\section{InTRODUCTION}

The set diffence between fuzzy sets which are defined by Zadeh in 1965, is given by using the concept of infimum[6]. However, there are many set difference conceptions between IFSs which are defined by Atanassov in 1983 because all implications and negations on IFSs theory generate different set differences between these sets[1].

Atanassov defined some fundamental operations on IFSs in [1],[2],[4] like as complement, $\cap, \cup,+, ., @, \&, \#, *$ New set differences can be defined between IVIFSs by the help of diversity of set differences between IFSs.

\section{On Intuitionistic Fuzzy Sets}

Some conceptions which are used in this paper, are given below,

The concept of fuzzy sets which is the extension of crisp sets by expanding the truth value set to unit interval [0.1], was introduced by Zadeh in 1965[6]. Let $X$ be a universal set. Then the function $\mu: X \rightarrow[0,1]$ is a fuzzy set over $X . \forall x \in X$, $\mu(x)$ is the membership degree of $\mathrm{x}$ and $1-\mu(x)$ is the non-membership degree of x. All fuzzy sets over $X$ are denoted by $F S(X)$.

Another extension of fuzzy sets is the concept of interval valued fuzzy sets that was defined by Sambuc in $1975[5]$. Let $X$ be a set and $A$ be interval valued fuzzy set. Then the function $M_{A}: X \rightarrow \wp([0,1]$ generates interval valued fuzzy set, where

Date: January, 2021.

2000 Mathematics Subject Classification. 05C38, 15A15, 05A15, 15A18.

Key words and phrases. Intuitionistic fuzzy sets, Interval valued intuitionistic fuzzy sets, Relations and operations over interval valued intuitionistic fuzzy sets. 
$\wp(x)$ is the power set of $X$. This means that for $x \in X, M_{A}(x)$ is a closed interval within $[0,1]$. All interval valued fuzzy sets over $X$ are denoted by $\operatorname{IVFS}(X)$.

Intuitionistic fuzzy sets which are the one of the extensions of fuzzy sets were defined by Atanassov in 1983[1]. Let $X$ be a universal set and A be an IFSs. Then $\mu_{A}(x)$ and $v_{A}(x)$ functions such that $0 \leq \mu_{A}(x)+v_{A}(x) \leq 1$ where $\mu_{A}: X \rightarrow[0.1]$ and $v_{A}: X \rightarrow[0,1]$ respectively are called membership and non-membership degree of each $x \in X$.It is important to point that the sum of membership and nonmembership degree of $\mathrm{x}$ over fuzzy set equals to 1 while this sum is less than 1 or equals to 1 over IFSs. All IFSs over $X$ are denoted by $\operatorname{IFS}(X)$.

The concept of interval valued intuitionistic fuzzy sets which is the extension both IFSs and interval valued fuzzy sets was defined by Atanassov and Gargov in 1989[3]. Let $X$ be a set and $A$ be interval valued intuitionistic fuzzy set. Then the functions $M_{A}: X \rightarrow \wp\left([0,1]\right.$ and $N_{A}: X \rightarrow \wp([0,1]$ generate interval valued intuitionistic fuzzy set, where $M_{A}(x) \subseteq[0,1]$ and $N_{A}(x) \subseteq[0,1]$ are closed interval such that $\sup M_{A}(x)+\sup N_{A}(x) \leq 1$. All interval valued intuitionistic fuzzy sets over $X$ are denoted by $\operatorname{IVIFS}(X)$.

In this paper, an interval valued fuzzy set $\mathrm{A}$ over universe set $\mathrm{X}$ is shown by $I V F S A$ and an interval valued intuitionistic fuzzy set $\mathrm{A}$ over universe set $\mathrm{X}$ is shown by IVIFS A.

Definition 1. [1] An intuitionistic fuzzy set (shortly, IFS) on a set $X$ is has the form

$$
A=\left\{\left\langle x, \mu_{A}(x), v_{A}(x) \mid x \in X\right\rangle\right\}
$$

where, $\mu_{A}(x)\left(\mu_{A}: X \rightarrow[0.1]\right)$ is called membership degree of $\mathrm{x}$ in A and $v_{A}(x)$ $\left(v_{A}: X \rightarrow[0,1]\right)$ is called non-membership degree of $\mathrm{x}$ in A. Also, $\mu_{A}$ and $v_{A}$ functions have inequality: $\forall x \in X, 0 \leq \mu_{A}(x)+v_{A}(x) \leq 1$

\section{On Interval Valued Intuitionistic Fuzzy Sets}

In this section, the definition of IVIFSs which is introduced by Atanassov and Gargov and the operations on IVIFSs are given.

Definition 2. [5] $X$ is a set. IVFS $A$ is

$$
A=\left\{\left\langle x, M_{A}(x)\right\rangle \mid x \in X\right\}
$$

the function; $M_{A}: X \rightarrow \wp[0,1]$ where $\wp(X)$ is the power set of $X . \forall x \in X$, $M_{A}(x)$ is a closed interval within $[0,1]$.

Definition 3. [3] $M_{A}(x) \subset[0,1]$ and $N_{A}(x) \subset[0,1]$ are closed interval and

$\forall x \in X, M_{A}: X \rightarrow \wp[0,1]$ and $N_{A}: X \rightarrow \wp[0,1]$ satisfy that, $\sup M_{A}(x)+$ $\sup N_{A}(x) \leq 1$

$$
A=\left\{\left\langle x, M_{A}(x), N_{A}(x)\right\rangle \mid x \in X\right\}
$$

is called interval valued intuitionistic fuzzy set over X. It is shortly denoted IVIFSs A

Some operations betwwen IVIFSs are given below.

Definition 4. [3] For any two IVIFSs $A$ and B, following relations are defined:

(1) $A \subset \square$, inf $B: \Leftrightarrow \forall x \in X, \inf M_{A}(x) \leq \inf M_{B}(x)$ 
(2) $A \subset \square$,sup $B: \Leftrightarrow \forall x \in X \sup M_{A}(x) \leq \sup M_{B}(x)$

(3) $A \subset \diamond$,inf $B: \Leftrightarrow \forall x \in X$, inf $N_{A}(x) \geq N_{B}(x)$

(4) $A \subset \diamond$,sup $B: \Leftrightarrow \forall x \in X, \inf N_{A}(x) \geq N_{B}(x)$

(5) $A \subset \square B: \Leftrightarrow A \subset \square$,inf and $A \subset \square$,sup $B$

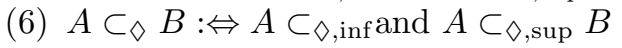

(7) $A \subset B: \Leftrightarrow A \subset \square B$ and $A \subset \diamond B$

(8) $A=B: \Leftrightarrow A \subset B$ and $B \subset A$

In this study, some operations between IVIFSs which are used to give the new set difference operation.

Definition 5. [3] For any two IVIFSs $A$ and $B$ the following operations are defined:

(1) $A^{c}=\left\{\left\langle x, N_{A}(x), M_{A}(x)\right\rangle \mid x \in X\right\}$

(2) $\left.A \cap B=\left\{\begin{array}{c}x,\left[\min \left\{\inf M_{A}(x), \inf M_{B}(x)\right\}, \min \left\{\sup M_{A}(x), \sup M_{B}(x)\right\}\right], \\ {\left[\max \left\{\inf N_{A}(x), \inf N_{B}(x)\right\}, \max \left\{\sup N_{A}(x), \sup N_{B}(x)\right\}\right]}\end{array}\right\rangle \mid x \in X\right\}$

(3) $\left.A \cup B=\left\{\begin{array}{c}x,\left[\max \left\{\inf M_{A}(x), \inf M_{B}(x)\right\}, \max \left\{\sup M_{A}(x), \sup M_{B}(x)\right\}\right], \\ {\left[\min \left\{\inf N_{A}(x), \inf N_{B}(x)\right\}, \min \left\{\sup N_{A}(x), \sup N_{B}(x)\right\}\right]}\end{array}\right\rangle \mid x \in X\right\}$

(4) $\left.A-\cap B=\left\{\begin{array}{c}x,\left[\min \left\{\inf M_{A}(x), \inf N_{B}(x)\right\}, \min \left\{\sup M_{A}(x), \sup N_{B}(x)\right\}\right], \\ {\left[\max \left\{\inf N_{A}(x), \inf M_{B}(x)\right\}, \max \left\{\sup N_{A}(x), \sup M_{B}(x)\right\}\right]}\end{array}\right\rangle \mid x \in X\right\}$

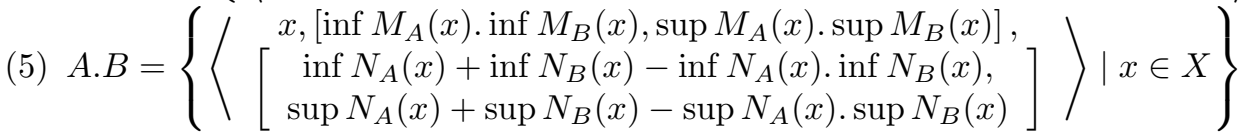

Theorem 1. [3] For every three IVIFS A, B and $C$,

(1) $A \cap B=B \cap A$

(2) $A \cup B=B \cup A$

(3) $A \cdot B=B \cdot A$

(4) $(A \cap B) \cap C=A \cap(B \cap C)$

(5) $(A \cup B) \cup C=A \cup(B \cup C)$

(6) $(A . B) . C=A .(B . C)$

(7) $(A \cap B) \cup C=(A \cup C) \cap(B \cup C)$

(8) $(A \cap B) . C=(A . C) \cap(B . C)$

(9) $(A \cup B) \cap C=(A \cap C) \cup(B \cap C)$

(10) $(A \cup B) . C=(A . C) \cup(B . C)$

The theorem that is given below is related to some properties of the concept of complement. Also, we examine these properties by using the new set difference.

Theorem 2. [3] For every IVIFS $A$ and $B$,

(1) $\left(A^{c} \cap B^{c}\right)^{c}=A \cup B$

(2) $\left(A^{c} \cup B^{c}\right)^{c}=A \cap B$

Definition 6. [3] There are some special sets on uncertain set theories. These special sets are null set and universel set on crisp set theory. Special sets on IVIFSs set theory are given below,

(1) $0^{*}=\{\langle x,[0,0],[1,1]\rangle \mid x \in X\}$

(2) $U^{*}=\{\langle x,[0,0],[0,0]\rangle \mid x \in X\}$

(3) $X^{*}=\{\langle x,[1,1],[0,0]\rangle \mid x \in X\}$

Remark 1. [3] It is clearly seen that: 


$$
0^{*} \subset U^{*} \subset X^{*}
$$

Remark 2. [3] $\forall I V I F S A$ :

$$
\begin{aligned}
& A \cap 0^{*}=A \cdot 0^{*}=0^{*} \\
& A \cup 0^{*}=A+0^{*}=0^{*}
\end{aligned}
$$

\section{The New Set Differences Operations Between IVIFSs}

In this section, we define new set difference between IVIFSs by using - operation. Accroding to these, the definition of $A-B$ is given below.

Definition 7. For any two IVIFSs $A$ and B, following operation are given:

$$
\left.A-B=\left\{\left\langle\begin{array}{c}
x,\left[\inf M_{A}(x) \cdot \inf N_{B}(x), \sup M_{A}(x) \cdot \sup N_{B}(x)\right], \\
\inf N_{A}(x)+\inf M_{B}(x)-\inf N_{A}(x) \cdot \inf M_{B}(x), \\
\sup N_{A}(x)+\sup M_{B}(x)-\sup N_{A}(x) \cdot \sup M_{B}(x)
\end{array}\right]\right\rangle \mid x \in X\right\}
$$

Proposition 1. For every IVIFSs $A$ and $B$,

$$
A-. B \in I V I F S(X)
$$

Proof. Arbitrary IVIFSs A and B are given,

$$
\left.A-B=\left\{\left\langle\begin{array}{c}
x,\left[\inf M_{A}(x) \cdot \inf N_{B}(x), \sup M_{A}(x) \cdot \sup N_{B}(x)\right], \\
\inf N_{A}(x)+\inf M_{B}(x)-\inf N_{A}(x) \cdot \inf M_{B}(x), \\
\sup N_{A}(x)+\sup M_{B}(x)-\sup N_{A}(x) \cdot \sup M_{B}(x)
\end{array}\right]\right\rangle \mid x \in X\right\}
$$

Now, we want to get that intervals are subsets of $[0,1]$.

$\inf M_{A}(x)$ and $\inf N_{B}(x) \in[0,1] \Rightarrow \inf M_{A}(x) . \inf N_{B}(x) \in[0,1]$

$\sup M_{A}(x)$ and $\sup N_{B}(x) \in[0,1] \Rightarrow \sup M_{A}(x) \cdot \sup N_{B}(x) \in[0,1]$

$\inf M_{A}(x) \leq \sup M_{A}(x)$ and $\inf N_{B}(x) \leq \sup N_{B}(x)$

$\Rightarrow \inf M_{A}(x) \cdot \inf N_{B}(x) \leq \sup M_{A}(x) \cdot \sup N_{B}(x)$

$\Rightarrow\left[\inf M_{A}(x) \cdot \inf N_{B}(x), \sup M_{A}(x) \cdot \sup N_{B}(x)\right] \subset[0,1]$

$\inf N_{A}(x)+\inf M_{B}(x) \geq \inf N_{A}(x) . \inf M_{B}(x) \Rightarrow$

$\inf N_{A}(x)+\inf M_{B}(x)-\inf N_{A}(x) \cdot \inf M_{B}(x) \geq 0$

Let inf $N_{A}(x)+\inf M_{B}(x)-\inf N_{A}(x) . \inf M_{B}(x)>1$. Then,

$\inf M_{B}(x)-\inf N_{A}(x) . \inf M_{B}(x)>1-\inf N_{A}(x) \Rightarrow$

$\inf M_{B}(x)\left(1-\inf N_{A}(x)\right)>1-\inf N_{A}(x)$

$\Rightarrow \inf M_{B}(x)>1$ This is a contradiction. Therefore,

$\inf N_{A}(x)+\inf M_{B}(x)-\inf N_{A}(x) . \inf M_{B}(x) \leq 1$

$\Rightarrow \inf N_{A}(x)+\inf M_{B}(x)-\inf N_{A}(x) . \inf M_{B}(x) \in[0,1]$

$\sup N_{A}(x)+\sup M_{B}(x) \geq \sup N_{A}(x) \cdot \sup M_{B}(x) \Rightarrow$ $\sup N_{A}(x)+\sup M_{B}(x)-\sup N_{A}(x) \cdot \sup M_{B}(x) \geq 0$

Let $\sup N_{A}(x)+\sup M_{B}(x)-\sup N_{A}(x) \cdot \sup M_{B}(x)>1$. Then, $\sup M_{B}(x)-\sup N_{A}(x) \cdot \sup M_{B}(x)>1-\sup N_{A}(x) \Rightarrow$ $\sup M_{B}(x)\left(1-\sup N_{A}(x)\right)>1-\sup N_{A}(x)$

$\Rightarrow \sup M_{B}(x)>1$ This is a contradiction. Therefore, $\sup N_{A}(x)+\sup M_{B}(x)-\sup N_{A}(x) \cdot \sup M_{B}(x) \leq 1$ $\Rightarrow \sup N_{A}(x)+\sup M_{B}(x)-\sup N_{A}(x) \cdot \sup M_{B}(x) \in[0,1]$ $\inf N_{A}(x) \leq \sup N_{A}(x) \Rightarrow 1-\sup N_{A}(x) \leq 1-\inf N_{A}(x)$ and 
$\inf M_{B}(x) \leq \sup M_{B}(x) \Rightarrow 1-\sup M_{B}(x) \leq 1-\inf M_{B}(x)$

$\Rightarrow\left(1-\sup N_{A}(x)\right)\left(1-\sup M_{B}(x)\right) \leq\left(1-\inf N_{A}(x)\right)\left(1-\inf M_{B}(x)\right)$

$\Rightarrow 1-\sup N_{A}(x)-\sup M_{B}(x)+\sup N_{A}(x) \cdot \sup M_{B}(x) \leq 1-\inf N_{A}(x)-\inf M_{B}(x)+$ $\inf N_{A}(x) . \inf M_{B}(x)$

$\Rightarrow-\sup N_{A}(x)-\sup M_{B}(x)+\sup N_{A}(x) \cdot \sup M_{B}(x) \leq-\inf N_{A}(x)-\inf M_{B}(x)+$ $\inf N_{A}(x) . \inf M_{B}(x)$

$\Rightarrow \sup N_{A}(x)+\sup M_{B}(x)-\sup N_{A}(x) \cdot \sup M_{B}(x) \geq \inf N_{A}(x)+\inf M_{B}(x)-$ $\inf N_{A}(x)$. inf $M_{B}(x)$

$\Rightarrow\left[\begin{array}{c}\inf N_{A}(x)+\inf M_{B}(x)-\inf N_{A}(x) \cdot \inf M_{B}(x), \\ \sup N_{A}(x)+\sup M_{B}(x)-\sup N_{A}(x) \cdot \sup M_{B}(x)\end{array}\right] \subset[0,1]$

Now, we show that the sum of the supremums of intervals is equal to 1 or less than 1.

$$
\begin{aligned}
& \sup M_{A}(x) \cdot \sup N_{B}(x)+\sup N_{A}(x)+\sup M_{B}(x)-\sup N_{A}(x) \cdot \sup M_{B}(x) \\
& =\sup M_{A}(x) \cdot \sup N_{B}(x)+\sup N_{A}(x)+\sup M_{B}(x)-\sup N_{A}(x) \cdot \sup M_{B}(x)+1-1 \\
& =\sup M_{A}(x) \cdot \sup N_{B}(x)+\sup N_{A}(x)\left(1-\sup M_{B}(x)\right)-\left(1-\sup M_{B}(x)\right)+1 \\
& =\sup M_{A}(x) \cdot \sup N_{B}(x)+\left(\sup N_{A}(x)-1\right) \cdot\left(1-\sup M_{B}(x)\right)+1 \\
& =\sup M_{A}(x) \cdot \sup N_{B}(x)-\left(1-\sup N_{A}(x)\right) \cdot\left(1-\sup M_{B}(x)\right)+1 \\
& \quad \text { We know that; } \\
& \left\{\begin{array}{c}
\sup M_{A}(x) \leq 1-\sup N_{A}(x) \text { and } \sup N_{B}(x) \leq 1-\sup M_{B}(x) \\
\Rightarrow \sup M_{A}(x) \cdot \sup N_{B}(x) \leq\left(1-\sup N_{A}(x)\right) \cdot\left(1-\sup M_{B}(x)\right) \\
\Rightarrow \sup M_{A}(x) \cdot \sup N_{B}(x)-\left(1-\sup N_{A}(x)\right) \cdot\left(1-\sup M_{B}(x)\right) \leq 0
\end{array}\right\} \\
& \Rightarrow \sup M_{A}(x) \cdot \sup N_{B}(x)-\left(1-\sup N_{A}(x) \cdot\left(1-\sup M_{B}(x)\right)+1 \leq 1\right. \\
& \Rightarrow \sup M_{A}(x) \cdot \sup N_{B}(x)+\sup N_{A}(x)+\sup M_{B}(x)-\sup N_{A}(x) \cdot \sup M_{B}(x) \leq 1 \\
& \text { We get that; } A-\cdot B \in I V I F S(X)
\end{aligned}
$$

Proposition 2. For every IVIFSs $A$ and $B$,

$$
A-B \subset A
$$

Proof. Arbitrary IVIFSs A and B are given, $\inf M_{A}(x) . \inf N_{B}(x) \leq \inf M_{A}(x), \sup M_{A}(x) \cdot \sup N_{B}(x) \leq \sup M_{A}(x)$ and Let $\inf N_{A}(x)+\inf M_{B}(x)-\inf N_{A}(x) . \inf M_{B}(x)<\inf N_{A}(x)$ then, $\inf M_{B}(x)-\inf N_{A}(x) . \inf M_{B}(x)<0$

$\Rightarrow \inf M_{B}(x)\left(1-\inf N_{A}(x)\right)<0$

$\Rightarrow 1-\inf N_{A}(x)<0 \Rightarrow \inf N_{A}(x)>1$ This a contradiction. Therefore, $\inf N_{A}(x)+\inf M_{B}(x)-\inf N_{A}(x) \cdot \inf M_{B}(x) \geq \inf N_{A}(x)$

Let $\sup N_{A}(x)+\sup M_{B}(x)-\sup N_{A}(x) . \sup M_{B}(x)<\sup N_{A}(x)$ then, $\sup M_{B}(x)-\sup N_{A}(x) \cdot \sup M_{B}(x)<0$

$\Rightarrow \sup M_{B}(x)\left(1-\sup N_{A}(x)\right)<0$

$\Rightarrow 1-\sup N_{A}(x)<0 \Rightarrow \sup N_{A}(x)>1$ This is a contradiction. Therefore, $\sup N_{A}(x)+\sup M_{B}(x)-\sup N_{A}(x) \cdot \sup M_{B}(x) \geq \sup N_{A}(x)$

Example 1. For two IVIFSs $A$ and $B$;

$$
A-\cap B \neq A-. B
$$

Solution 1. Let universet set $X$ be:

$X=\{a, b, c, d\}$

IVIFSs $\mathrm{A}$ and $\mathrm{B}$ are:

$A=\{\langle a,[0,0.5],[0.2,0.4]\rangle,\langle b,[0.1,0.3],[0.4,0.5]\rangle,\langle c,[0.2,0.7],[0,0.1]\rangle,\langle d,[0.6,0.8],[0.1,0.2]\rangle\}$ 
$B=\{\langle a,[0.1,0.3],[0.5,0.7]\rangle,\langle b,[0.3,0.6],[0.2,0.3]\rangle,\langle c,[0.2,0.6],[0.3,0.4]\rangle,\langle d,[0.4,0.5],[0.1,0.3]\rangle\}$ below sets are:

$$
\begin{aligned}
& A-\cap B=\left\{\begin{array}{c}
\langle a,[\min \{0,0.5\}, \min \{0.5,0.7\}],[\max \{0.2,0.1\}, \max \{0.4,0.3\}]\rangle, \\
\langle b,[\min \{0.1,0.2\}, \min \{0.3,0.3\}],[\max \{0.4,0.3\}, \max \{0.5,0.6\}]\rangle \\
\langle c,[\min \{0.2,0.3\}, \min \{0.7,0.4\}],[\max \{0,0.2\}, \max \{0.1,0.6\}]\rangle \\
\langle d,[\min \{0.6,0.1\}, \min \{0.8,0.3\}],[\max \{0.1,0.4\}, \max \{0.2,0.5\}]\rangle
\end{array}\right\} \\
& \Rightarrow A-\cap B=\left\{\begin{array}{c}
\langle a,[0,0.5],[0.2,0.4]\rangle,\langle b,[0.1,0.3],[0.4,0.6]\rangle, \\
\langle c,[0.2,0.4],[0.2,0.6]\rangle,\langle d,[0.1,0.3],[0.4,0.5]\rangle
\end{array}\right\}
\end{aligned}
$$

and

$$
\begin{aligned}
& A-. B=\left\{\begin{array}{c}
\langle a,[0 .(0.5),(0.5) \cdot(0.7)],[0.2+0.1-(0.2) \cdot(0.1), 0.4+0.3-(0.4) .(0.3)]\rangle \\
\langle b,[(0.1) \cdot(0.2),(0.3) \cdot(0.3)],[0.4+0.3-(0.4) \cdot(0.3), 0.5+0.6-(0.5) \cdot(0.6)]\rangle \\
\langle c,[(0.2) \cdot(0.3),(0.7) \cdot(0.4)],[0+0.2-0 .(0.2), 0.1+0.6-(0.1) \cdot(0.6)]\rangle \\
\langle d,[(0.6) \cdot(0.1),(0.8) \cdot(0.3)],[0.1+0.4-(0.1) \cdot(0.4), 0.2+0.5-(0.2) .(0.5)]\rangle
\end{array}\right\} \\
& \Rightarrow A-. B=\left\{\begin{array}{l}
\langle a,[0,0.35],[0.28,0.58]\rangle,\langle b,[0.02,0.09],[0.58,0.8]\rangle, \\
\langle c,[0.06,0.28],[0.2,0.64]\rangle,\langle d,[0.06,0.24],[0.46,0.6]\rangle
\end{array}\right\}
\end{aligned}
$$

It is clearly seen that; $A-\cap B \neq A-B$

The result of above discussions is that $A-B$ is IVIFs. This set difference is not equal to $A-\cap B$. Therefore, $X-. A$ is the complement of IVIFSs A which is determined by - operation. It is shortly shown $A^{c}$.

Proposition 3. For every IVIFSs $A$ and $B$,

$A-B \subset A-\cap B$

Proof. Arbitrary IVIFSs A and B are given,

$\inf M_{A}(x) . \inf N_{B}(x) \leq \inf M_{A}(x)$ and $\inf N_{B}(x) \Rightarrow$

$\inf M_{A}(x) \cdot \inf N_{B}(x) \leq \min \left\{\inf M_{A}(x), \inf N_{B}(x)\right\}$

$\sup M_{A}(x) \cdot \sup N_{B}(x) \leq \sup M_{A}(x)$ and $\sup N_{B}(x) \Rightarrow$

$\sup M_{A}(x) \cdot \sup N_{B}(x) \leq \min \left\{\sup M_{A}(x), \sup N_{B}(x)\right\}$

and $\left.\begin{array}{rl} & \inf N_{A}(x)+\inf M_{B}(x)-\inf N_{A}(x) \cdot \inf M_{B}(x) \geq \inf N_{A}(x) \text { and } \inf M_{B}(x) \\ \Rightarrow & \inf N_{A}(x)+\inf M_{B}(x)-\inf N_{A}(x) \cdot \inf M_{B}(x) \geq \max \left\{\inf N_{A}(x), \inf M_{B}(x)\right\}\end{array}\right\}$
$\left\{\begin{array}{c}\quad \sup N_{A}(x)+\sup M_{B}(x)-\sup N_{A}(x) \cdot \sup M_{B}(x) \geq \sup N_{A}(x) \text { and } \sup M_{B}(x) \\ \Rightarrow \sup N_{A}(x)+\sup M_{B}(x)-\sup N_{A}(x) \cdot \sup M_{B}(x) \geq \max \left\{\sup N_{A}(x), \sup M_{B}(x)\right\}\end{array}\right\}$
$\Rightarrow A-B \subset A-\cap B$

The normal complement of special sets are the same with - operation

Corollary 1. For three special IVIFSs,

(1) $\left(0^{*}\right)^{c} .=X^{*}$ and $\left(\left(0^{*}\right)^{c} \cdot\right)^{c} \cdot=0^{*}$

(2) $\left(U^{*}\right)^{c}=U^{*}$ and $\left(\left(U^{*}\right)^{c} \cdot\right)^{c} .=U^{*}$

(3) $\left(X^{*}\right)^{c}$. $=0^{*}$ and $\left(\left(X^{*}\right)^{c} \cdot\right)^{c .}=X^{*}$

(4) For every IVIFSs $\mathrm{A}, A^{c} .=A^{c}$ and $\left(A^{c} \cdot\right)^{c} .=A$

In below corollary, the normal set differences between special sets are the same between IVIFSs

Corollary 2. For three special IVIFSs,

(1) $X^{*}-0^{*}=X^{*}$

(2) $X^{*}-U^{*}=U^{*}$

(3) $0^{*}-. X^{*}=0^{*}$

(4) $0^{*}-U^{*}=0^{*}$ 
(5) $U^{*}-. X^{*}=0^{*}$

(6) $U^{*}-.0^{*}=U^{*}$

De Morgan Laws are given below.

Proposition 4. For every IVIFSs $A$ and $B$,

(1) $(A \cap B)^{c} .=A^{c} \cdot \cup B^{c .}$

(2) $(A \cup B)^{c} \cdot=A^{c} \cdot \cap B^{c .}$

Proof. Arbitrary IVIFSs A and B are given,

1. $(A \cap B)^{c}=\left\{\left\langle\begin{array}{c}x,\left[1 \cdot \max \left\{\inf N_{A}(x), \inf N_{B}(x)\right\}, 1 \cdot \max \left\{\sup N_{A}(x), \sup N_{B}(x)\right\}\right], \\ 0+\min \left\{\inf M_{A}(x), \inf M_{B}(x)\right\}-0 \cdot \min \left\{\inf M_{A}(x), \inf M_{B}(x)\right\}, \\ 0+\min \left\{\sup M_{A}(x), \sup M_{B}(x)\right\}-0+\min \left\{\sup M_{A}(x), \sup M_{B}(x)\right\}\end{array}\right] \mid x \in X\right\}$

$\left.\Rightarrow(A \cap B)^{c} \cdot=\left\{\begin{array}{c}x,\left[\max \left\{\inf N_{A}(x), \inf N_{B}(x)\right\}, \max \left\{\sup N_{A}(x), \sup N_{B}(x)\right\}\right], \\ {\left[\min \left\{\inf M_{A}(x), \inf M_{B}(x)\right\}, \min \left\{\sup M_{A}(x), \sup M_{B}(x)\right\}\right]}\end{array}\right\rangle \mid x \in X\right\}$

$=A^{c} \cdot \cup B^{c .}$

2. $(A \cup B)^{c} \cdot=\left\{\left\langle\begin{array}{c}x,\left[1 \cdot \min \left\{\inf N_{A}(x), \inf N_{B}(x)\right\}, 1 \cdot \min \left\{\sup N_{A}(x), \sup N_{B}(x)\right\}\right], \\ 0+\max \left\{\inf M_{A}(x), \inf M_{B}(x)\right\}-0 \cdot \max \left\{\inf M_{A}(x), \inf M_{B}(x)\right\}, \\ 0+\max \left\{\sup M_{A}(x), \sup M_{B}(x)\right\}-0 \cdot \max \left\{\sup M_{A}(x), \sup M_{B}(x)\right\}\end{array}\right] \mid x \in X\right\}$ $\left.\Rightarrow(A \cup B)^{c}=\left\{\begin{array}{c}x,\left[\min \left\{\inf N_{A}(x), \inf N_{B}(x)\right\}, \min \left\{\sup N_{A}(x), \sup N_{B}(x)\right\}\right], \\ {\left[\max \left\{\inf M_{A}(x), \inf M_{B}(x)\right\}, \max \left\{\sup M_{A}(x), \sup M_{B}(x)\right\}\right]}\end{array}\right\rangle \mid x \in X\right\}$

$=A^{c} \cdot \cap B^{c}$.

In this below proposition, it is the same as crisp set.

Proposition 5. For every IVIFSs $A$. B and $C$,

(1) $A-.(B \cup C)=(A-. B) \cap(A-. C)$

(2) $A-.(B \cap C)=(A-. B) \cup(A-. C)$

Proof. Arbitrary IVIFSs A . B and C are given,

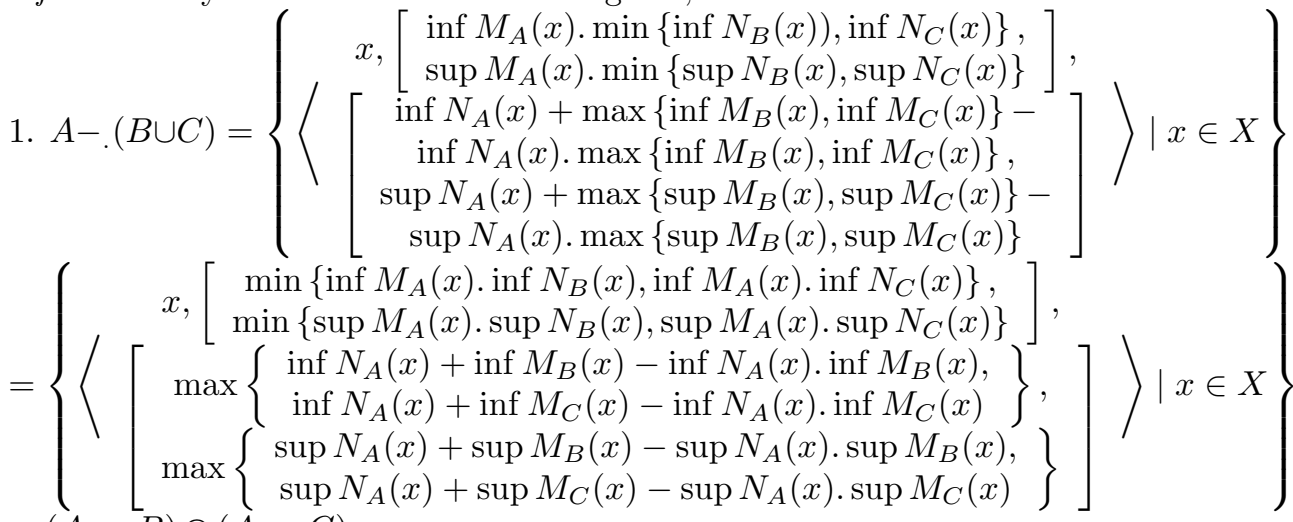

$=(A-. B) \cap(A-. C)$

2. $\left.A-.(B \cap C)=\left\{\left\langle\begin{array}{c}x,\left[\begin{array}{c}\left.\inf M_{A}(x) \cdot \max \left\{\inf N_{B}(x)\right), \inf N_{C}(x)\right\}, \\ \sup M_{A}(x) \cdot \max \left\{\sup N_{B}(x), \sup N_{C}(x)\right\}\end{array}\right], \\ \inf N_{A}(x)+\min \left\{\inf M_{B}(x), \inf M_{C}(x)\right\}- \\ \inf N_{A}(x) \cdot \min \left\{\inf M_{B}(x), \inf M_{C}(x)\right\}, \\ \left.\sup N_{A}(x)+\min \left\{\sup M_{B}(x)\right), \sup M_{C}(x)\right\}- \\ \left.\left.\sup N_{A}(x) \cdot \min \left\{\sup M_{B}(x)\right), \sup M_{C}(x)\right\}\right)\end{array}\right]\right\rangle \mid X\right\}$ 


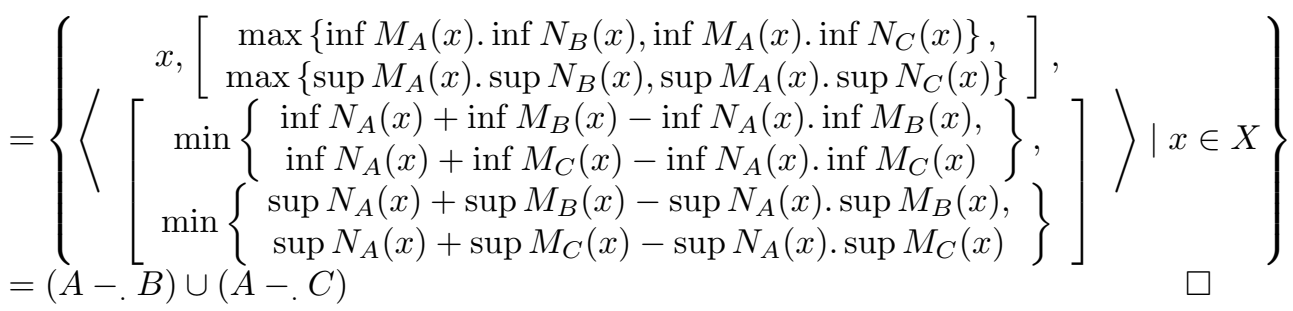

Proposition 6. For every IVIFSs $A . B$ and $C$,

(1) $(A \cap B)-. C=(A-. C) \cap(B-. C)$

(2) $(A \cup B)-. C=(A-. C) \cup(B-. C)$

Proof. Arbitrary IVIFSs A . B and C are given,

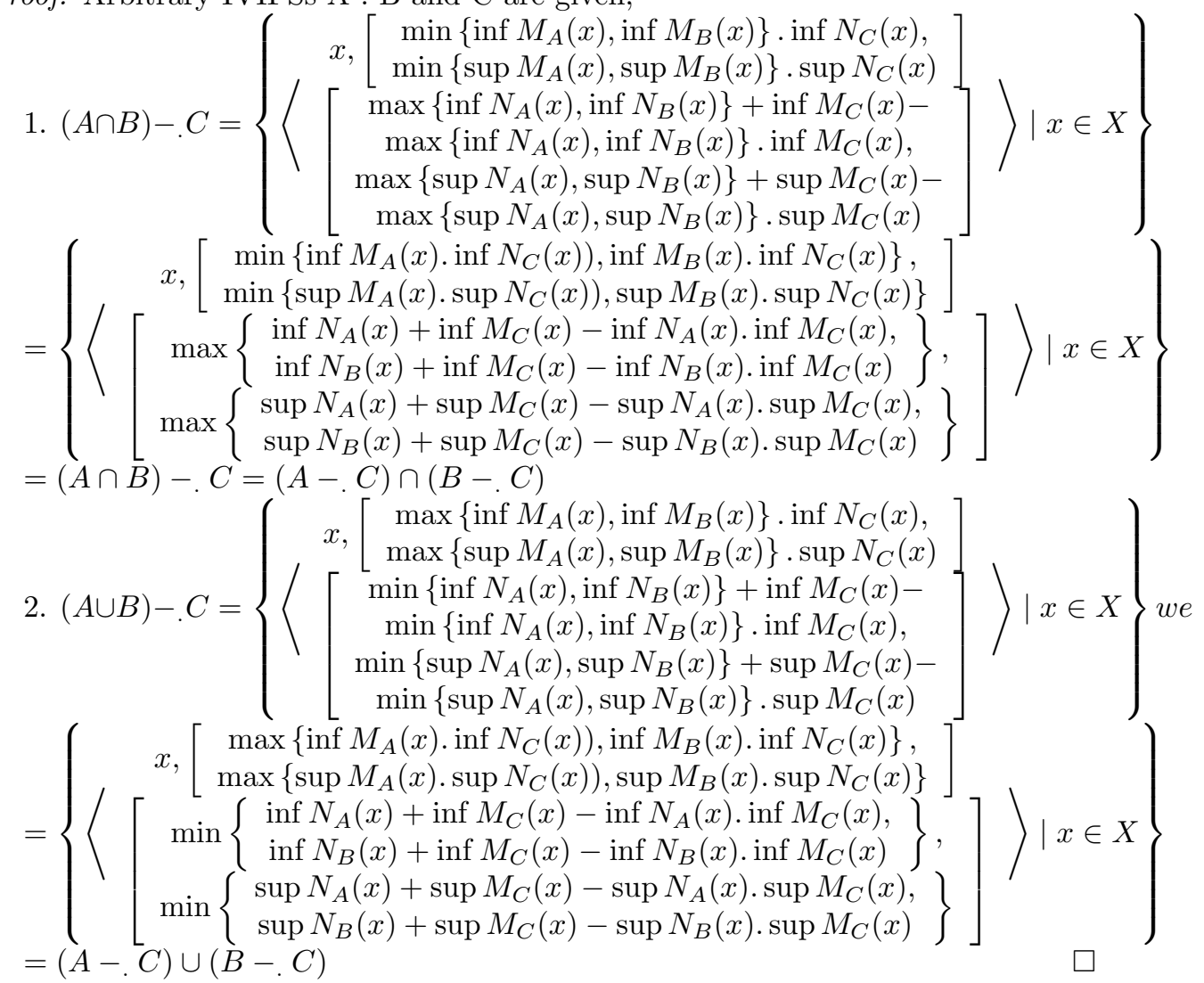

Corollary 3. For every IVIFSs $A$ and $B$,

$(A-. B) \cup A=A$

Proposition 7. For every IVIFSs $A, B$ and $C$,

$C \subset B \Rightarrow A-B \subset A-C$

Proof. Arbitrary IVIFSs A and B are given,

$\inf M_{A}(x) \cdot \inf N_{B}(x) \leq \inf M_{A}(x) . \inf N_{C}(x)$ and $\sup M_{A}(x) \cdot \sup N_{B}(x) \leq \sup M_{A}(x) \cdot \sup N_{C}(x)$ Let $\inf N_{A}(x)+\inf M_{B}(x)-\inf N_{A}(x) \cdot \inf M_{B}(x)<\inf N_{A}(x)+\inf M_{C}(x)-$ $\inf N_{A}(x) . \inf M_{C}(x)$ then, 
$\inf M_{B}(x)\left(1-\inf N_{A}(x)\right)<\inf M_{C}(x)\left(1-\inf N_{A}(x)\right) \Rightarrow \inf M_{B}(x)<\inf M_{C}(x)$ This is a contradiction. Therefore,

$\Rightarrow \inf N_{A}(x)+\inf M_{B}(x)-\inf N_{A}(x) . \inf M_{B}(x) \geq \inf N_{A}(x)+\inf M_{C}(x)-$ $\inf N_{A}(x) . \inf M_{C}(x)$

Let $\sup N_{A}(x)+\sup M_{B}(x)-\sup N_{A}(x) \cdot \sup M_{B}(x)<\sup N_{A}(x)+\sup M_{C}(x)-$ $\sup N_{A}(x) . \sup M_{C}(x)$ then,

$\sup M_{B}(x)\left(1-\sup N_{A}(x)\right)<\sup M_{C}(x)\left(1-\sup N_{A}(x)\right) \Rightarrow \sup M_{B}(x)<\sup M_{C}(x)$

This is a contradiction. Therefore,

$\Rightarrow \sup N_{A}(x)+\sup M_{B}(x)-\sup N_{A}(x) \cdot \sup M_{B}(x) \geq \sup N_{A}(x)+\sup M_{C}(x)-$ $\sup N_{A}(x) \cdot \sup M_{C}(x)$

We get that; $\Rightarrow A-. B \subset A-. C$

\section{REFERENCES}

[1] K. T. Atanassov, Intuitionistic Fuzzy Sets, VII ITKR's Session, Sofia, June 1983 (deposed in Central Sci.-Techn. Library of Bulg. Acad. Of Sci. No. 1697184 (in Bulgaria) 1983.

[2] K. T. Atanassov, Intuitionistic Fuzzy Sets, Fuzzy Sets and Systems 20 (1986) 87-96.

[3] K. Atanassov and G. Gargov, Interval valued intuitionistic fuzzy sets, Fuzzy Sets and Systems 31 (1989) 343-349.

[4] K. T. Atanassov, Intuitionisitc Fuzzy Sets, Phiysica-Verlag, Heidelberg, New York 1999.

[5] R. Sambuc, Functions $\phi$-flous.Aplication a l'aide au diagnostic en pathologie thyrodene, Thèse, Universite de Marseiile (1975).

[6] L. A. Zadeh, Fuzzy Sets, Information and Control (8) (1965) 338-353.

Vocational School of Technical Sciences, Mersin University

Email address: arif.bal.math@gmail.com

Department of Mathematics, Science and Literature Faculty, Mersin University

Email address: gcuvalcioglu@gmail.com 